\title{
Effect of Magnetic Field on the Magnetic Domain Structure of MnAs Film on GaAs(001)
}

\author{
J. B. Kim ${ }^{1}$, Y. P. Lee ${ }^{1}$, K. S. Ryu ${ }^{2}$, S. C. Shin ${ }^{2}$, H. Akinaga ${ }^{3}$, and K. W. Kim ${ }^{4}$ \\ ${ }^{1}$ Quantum Photonic Science Research Center and Department of Physics, Hanyang University, Seoul 133-791, Korea \\ ${ }^{2}$ Department of Physics and Center for Nanospinics of Spintronic Materials, \\ Korea Advanced Institute of Science and Technology, Daejeon 305-701, Korea \\ ${ }^{3}$ Nanotechnology Research Institute, National Institute of Advanced Industrial Science and Technology, Ibaraki 305-8562, Japan \\ ${ }^{4}$ Department of Physics, Sunmoon University, Asan 336-708, Korea
}

\begin{abstract}
We have observed the magnetic domain evolution under an external magnetic field in an epitaxial MnAs film on GaAs(001) by magnetic force microscopy. Owing to the strain involved, the ferromagnetic $\alpha$-MnAs and the paramagnetic $\beta$-MnAs phases coexist as self-aligned stripes at room temperature. It was found that a complex magnetic domain structure appeared in the ferromagnetic $\alpha$-phase region at the demagnetized state $(H=0 \mathrm{Oe})$. As the magnetic field increased, the magnetic domain structure was gradually changed, and reached a completely saturated state at $H=600$ Oe. Especially, at $H=300$ Oe, the observed magnetic domain wall distribution is well matched with the topographical shape.
\end{abstract}

Index Terms-Magnetic domains, magnetic force microscopy, magnetic thin films.

\section{INTRODUCTION}

$\mathbf{E}$ PITAXIAL MnAs on GaAs is one of the promising ferromagnetic/semiconductor hybrid systems for future spintronic applications [1]. A key to the success is the epitaxial growth of ferromagnet-semiconductor heterostructures with well-ordered interfaces, which allow the controlled spin injection from the ferromagnetic layer into the semiconductor. In spite of a very large and anisotropic lattice misfit, MnAs can be epitaxially grown on $\mathrm{GaAs}(001)$ in good quality [2]. While the bulk MnAs shows the first-order phase transition from the ferromagnetic, hexagonal $\alpha$-phase to the paramagnetic, orthorhombic $\beta$-phase at $313 \mathrm{~K}, \alpha-\mathrm{MnAs}$ and $\beta$-MnAs coexist in MnAs films epitaxially grown on $\mathrm{GaAs}(001)$ over a certain temperature range via the strain stabilization [3]-[7]. It is well known that the coexisting $\alpha-\mathrm{MnAs}$ and $\beta-\mathrm{MnAs}$ phases form a self-aligned stripe structure with varying periodicity depending on the film thickness. It makes this material system quite useful in spintronic applications. Therefore, the micromagnetic structure of MnAs film is important for the fundamental understanding on the interplay between elastic and magnetic structures and for its application to spin-injection device [8].

In the past few years, several groups have reported the submicron magnetic domain structure of MnAs films on $\mathrm{GaAs}(001)$ by magnetic force microscopy (MFM) measurements and simulations [9]. The temperature-dependent atomic force microscopy (AFM) and MFM show that the morphology and the structure of films are strongly correlated with the magnetic structure [3]. Low-energy electron microscopy (LEEM) and $\mathrm{X}$-ray magnetic-circular-dichroism photo emission electron microscopy (XMCDPEEM), which allows a direct mapping of the in-plane magnetization, were combined to investigate the micromagnetic properties with a high lateral resolution [4].

Digital Object Identifier 10.1109/TMAG.2006.878404
By analyzing the self-organized array of ferromagnetic stripes, the thickness dependence of the magnetic properties of MnAs films on $\mathrm{GaAs}(001)$ and $\mathrm{GaAs}(113)$ could be explained [10].

However, most of the previous studies have been concentrated only on the micromagnetic structure at a certain external magnetic field, and the magnetic domain evolution, by varying the external magnetic field, is not fully understood yet. In this paper, we report a systematic MFM/AFM observation on the $\mathrm{MnAs} / \mathrm{GaAs}(001)$ system by varying the applied magnetic field.

\section{EXPERIMENTAL WORK}

A 250-nm-thick MnAs film was epitaxially grown on a GaAs(001) substrate in a molecular-beam epitaxy system. Ahead of the growth of MnAs, the substrate was heated up to about $600{ }^{\circ} \mathrm{C}$ in the flux of $\mathrm{As}_{4}$ for thermal cleaning, followed by the deposition of a 350-nm-thick, undoped GaAs buffer layer. Subsequently, the MnAs film was grown at $270{ }^{\circ} \mathrm{C}$ with a growth rate of about $0.05 \mathrm{~nm} / \mathrm{s}$. The detailed growth conditions can be found elsewhere [11].

The magnetic properties of $\mathrm{MnAs} / \mathrm{GaAs}(001)$ film was investigated by using a superconducting-quantum-interference-device magnetometer (SQUID) (Quantum Design, MPMS-XL). The surface morphology and the magnetic domain structures of films were examined using a AFM/MFM system (PSIA, XE-100). The AFM/MFM was operated in the noncontact lift mode, which detects the change in the phase of the resonant frequency relative to the drive frequency. The tip scans the sample in the noncontact mode to obtain the surface morphology. The scan is repeated at a constant height above the surface so that the magnetic and the topographic signals are well separated. The magnetic cantilevers were purchased from Nanosensors. The cantilevers are coated with a cobalt alloy (40 $\mathrm{nm}$ thick) on the tip side and aluminum (30 $\mathrm{nm}$ thick) on the detector side. The tip radius is typically less than $50 \mathrm{~nm}$. In our measurement configuration, the MFM images were obtained using the interleave mode at a lift scan height of $50 \mathrm{~nm}$. The 


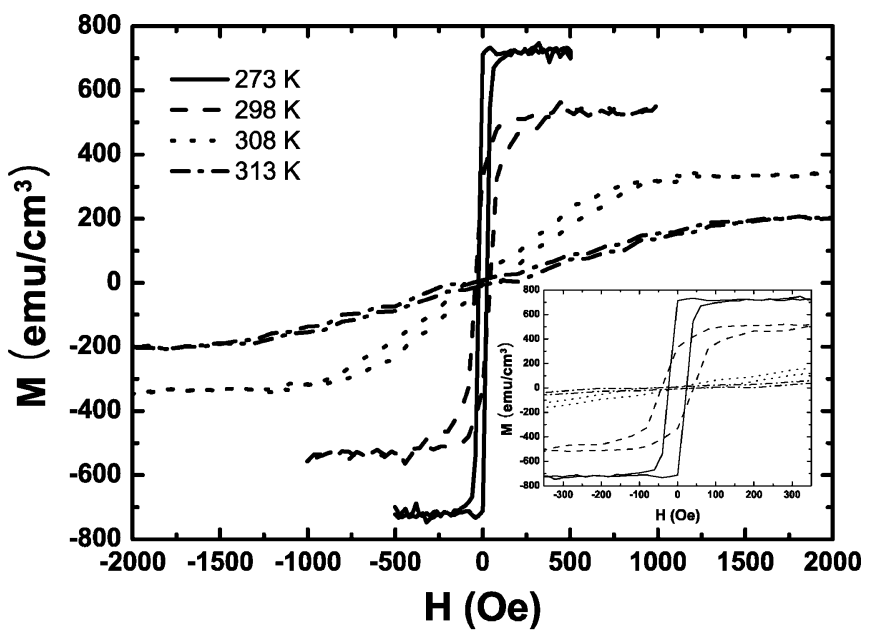

Fig. 1. Hysteresis loops at several temperatures by using SQUID in a magnetic field applied along the easy axis of film magnetization. The inset shows an expended view along $\mathrm{H}$.

contrast of the MFM images illustrates the interplay between the stray field above the film surface and the MFM tip. This AFM/MFM system was equipped with a special electromagnet, which allows us to investigate the evolutions of magnetic domains under an external in-plane magnetic field (up to $600 \mathrm{Oe}$ ).

\section{RESULTS AND DISCUSSION}

The temperature-dependent X-ray diffraction (XRD) measurement (not shown here), where the sample temperature was controlled by a radiative heating stage with an accuracy of $1 \mathrm{~K}$ in the temperature range of $293 \mathrm{~K}-318 \mathrm{~K}$, revealed that the peaks corresponding to $\alpha-\mathrm{MnAs}$ and $\beta-\mathrm{MnAs}$, appear at $293 \mathrm{~K}$ and $318 \mathrm{~K}$, respectively. A double-peak structure consisting of these two peaks was observed in the intermediate temperature range with a systematic variation of the relative intensities of the two peaks with temperature; the $\beta-\mathrm{MnAs}$ peak intensity was increased with increasing temperature as expected. It has been shown [11] that the sample had an in-plane magnetic anisotropy with an easy axis along the MnAs[11 $\overline{2} 0]$ and a hard axis along the MnAs [0001] in the film plane. The out-of-plane direction of MnAs[1100] was an intermediate axis.

Fig. 1 presents the magnetic hysteresis loops of MnAs/GaAs(001) film measured by using a SQUID magnetometer at several temperatures around the phase transition. External magnetic field was applied along the easy axis of the sample. At low temperatures, where the $\alpha$-phase is dominant, the magnetization of the sample is easily saturated with a high saturation magnetization and a small coercivity. The ratio of the remanent magnetization to the saturation magnetization $M_{r} / M_{s}$ is close to unity. This indicates that the reversible part of the magnetization is very small, as expected for coupled ferromagnetic stripes [12]. As the temperature increases, the magnetic field required for saturation increases and the saturation magnetization decreases. The reduction of saturation magnetization reflects the decrease of the relative amount of the ferromagnetic phase at higher temperatures. Reduction

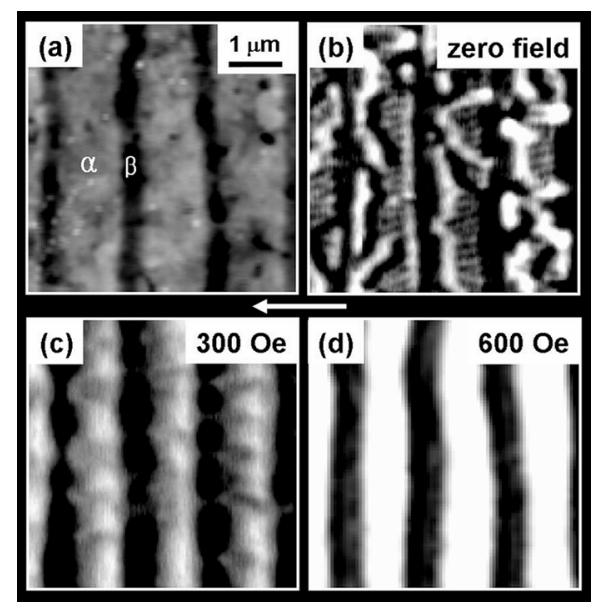

Fig. 2. (a) AFM and (b)-(d) MFM images at room temperature for 250-nm-thick MnAs film on GaAs(001). All the images were taken from the same scan area $\left(5 \times 5 \mu \mathrm{m}^{2}\right)$ and position. The magnetic field is applied along the easy axis of magnetization as indicated by the white arrow.

of the $M_{r} / M_{s}$ ratio below 1 reflects the decreased coupling between the ferromagnetic stripes at higher temperatures.

The AFM and the MFM images were taken from the same $5 \times 5 \mu \mathrm{m}^{2}$ scan area and position at room temperature. Fig. 2(b)-(d) shows a series of MFM images acquired at different field values as indicated in each image. For the MFM measurements, an external magnetic field was applied along the magnetic easy axis, which is indicated by a white arrow in Fig. 2. By comparing the AFM and the MFM images, it is clear that the topography and the magnetic contrast are well separated; while no change was observed in the topographic image, the magnetic domain structures gradually changed as the external magnetic field was varied. The AFM image clearly shows a stripe pattern which corresponds to the alternating ferromagnetic $\alpha$ - and paramagnetic $\beta$-phase regions with a periodicity of about $1.5 \mu \mathrm{m}$ [see Fig. 2(d)]. The demagnetized state was prepared by heating the film above the phase-transition temperature until it was completely in its $\beta$-phase and then cooled down to room temperature in zero field. In the demagnetized state, 250-nm-thick MnAs film on GaAs(001) exhibits rather complex magnetic domain patterns consisting of elongated, short zig-zag-shaped, and comb-like domains. It is common that domain walls (DWs) are not only directed perpendicular or parallel to the stripes but do occur under intermediate angles as well in MnAs/GaAs(001) system [13]. As the magnetic field increases, the complex magnetic domains gradually collapse with each other, and reach a completely saturated state at $H=600 \mathrm{Oe}$.

Especially at $H=300$ Oe, the observed magnetic DW distribution is well matched with the topological one [see Fig. 3(b)]. This fact reveals a strong correlation between the topological shape and magnetic domain structures. Since the specific magnetic domain structures inside the patterned films are closely related to the size and the shape anisotropy, and is determined by a competition between the exchange energy and the shape-dependent demagnetization energy, further studies, including the micromagnetic simulations and the investigations on the magnetic domain evolutions in films with varying thickness, are in progress. 


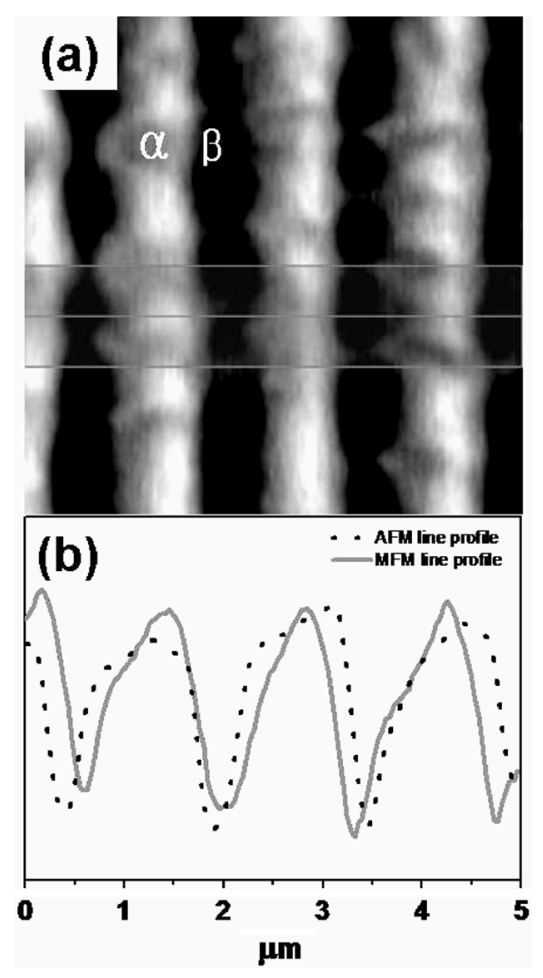

Fig. 3. (a) MFM image at at room temperature and $H=300$ Oe. (b) Line profiles of the MFM and AFM signal averaged across the stripe in the rectangular area defined in (a).

\section{CONCLUSION}

A 250-nm-thick MnAs film was epitaxially grown on a $\operatorname{GaAs}(001)$ substrate in a molecular-beam epitaxy system, and the magnetic-domain evolution under an external magnetic field was observed by magnetic force microscopy. Due to the strain involved, ferromagnetic $\alpha-\mathrm{MnAs}$ and paramagnetic $\beta-\mathrm{MnAs}$ phases coexist as stripes at room temperature. It was found that a complex magnetic domain structure appeared in the ferromagnetic $\alpha$-phase region at the demagnetized state $(H=0 \mathrm{Oe})$. As the magnetic field increased, the complex magnetic domain structure gradually changed, and reached a completely saturated state at $H=600$ Oe. Especially, at $H=300$ Oe, the observed DW distribution was well matched with the topographical shape.

\section{ACKNOWLEDGMENT}

This work was supported by the Korea Science and Engineering Foundation (KOSEF) through the Quantum Photonic Science Research Center at Hanyang University, Seoul, Korea, and by MOST, Korea.

\section{REFERENCES}

[1] G. A. Prinz, "Hybrid ferromagnetic-semiconductor structures," Science, vol. 250, no. 4984, pp. 1092-1097, Nov. 1990.

[2] M. Tanaka, J. P. Harbison, M. C. Park, Y. S. Park, T. Shin, and G. M. Rothberg, "Epitaxial ferromagnetic MnAs thin films grown by molecular-beam epitaxy on GaAs: Structure and magnetic properties," J. Appl. Phys., vol. 76, pp. 6278-6280, Nov. 1994.

[3] T. Plake, T. Hesjedal, J. Mohanty, M. Kästner, L. Däweritz, and K. H. Ploog, "Temperature-dependent magnetic force microscopy investigation of epitaxial MnAs films on GaAs(001)," Appl. Phys. Lett., vol. 82, pp. 2308-2310, Apr. 2003.

[4] E. Bauer, S. Cherifi, L. Däweritz, M. Kästner, S. Heun, and A. Locatelli, "Low-energy electron microscopy/X-ray magnetic circular dichroism photoemission electron microscopy study of epitaxial MnAs on GaAs," J. Vac. Sci. Technol. B, vol. 20, pp. 2539-2542, Nov. 2002.

[5] F. Schippan, G. Behme, L. Däweritz, K. H. Ploog, B. Dennis, K.-U. Neumann, and K. R. A. Ziebeck, "Magnetic structure of epitaxially grown MnAs on GaAs(001)," J. Appl. Phys., vol. 88, pp. 2766-2770, Sep. 2000.

[6] V. M. Kaganer, B. Jenichen, F. Schippan, W. Braun, L. Däweritz, and K. H. Ploog, "Strain-mediated phase coexistence in heteroepitaxial films," Phys. Rev. Lett., vol. 85, pp. 341-344, Sep. 2000.

[7] T. Plake, M. Ramsteiner, V. M. Kaganer, B. Jenichen, M. Kästner, L. Däweritz, and K. H. Ploog, "Periodic elastic domains of coexisting phases in epitaxial MnAs films on GaAs," Appl. Phys. Lett., vol. 80, pp. 2523-2525, Apr. 2002.

[8] M. Ramsteiner, H. Y. Hao, A. Kawaharazuka, H. J. Zhu, M. Kästner R. Hey, L. Däweritz, H. T. Grahn, and K. H. Ploog, "Electrical spin injection from ferromagnetic MnAs metal layers into GaAs," Phys. Rev. $B$, vol. 66, Aug. 2002. 081304(R).

[9] R. Engel-Herbert, J. Mohanty, A. Ney, T. Hesjedal, L. Däweritz, and K. H. Ploog, "Understanding the submicron domain structure of MnAs thin films on GaAs(001): Magnetic force microscopy measurements and simulations," Appl. Phys. Lett., vol. 84, pp. 1132-1134, Feb. 2004.

[10] L. Däweritz, L. Wan, B. Jenichen, C. Herrmann, J. Mohanty, A. Trampert, and K. H. Ploog, "Thickness dependence of the magnetic properties of MnAs films on $\mathrm{GaAs}(001)$ and $\mathrm{GaAs}(113)$ : Role of a natural array of ferromagnetic stripes," J. Appl. Phys., vol. 96, pp. 5056-5062, Nov. 2004.

[11] K.-S. Ryu, D.-H. Kim, S.-C. Shin, and H. Akinaga, "Real-time direct observation of asymmetric magnetization reversal in exchange-biased single-layer systems," Phys. Rev. B, vol. 71, Apr. 2005. 155308.

[12] R. Engel-Herbert, T. Hesjedal, J. Mohanty, D. M. Schaadt, and K. H Ploog, "Field dependence of micromagnetic domain patterns in MnAs films," J. Appl. Phys., vol. 98, Sep. 2005. 063909.

[13] A. Ney, T. Hesjedal, and K. H. Ploog, "Competing magnetic interactions in MnAs studied via thin film domain pattern analysis," Phys. Rev. B, vol. 72, Dec. 2005. 212412.

Manuscript received March 10, 2006 (e-mail: yplee@hanyang.ac.kr). 